\title{
Interference Aware MMSE Equalization for MIMO TxAA
}

\author{
M. Wrulich, C. Mehlführer and M. Rupp \\ Institute of Communications and Radio-Frequency Engineering, Vienna University of Technology \\ Gußhausstraße 25/389, 1040 Vienna, Austria \\ Email: \{mwrulich,chmehl,mrupp\}@nt.tuwien.ac.at \\ Web: http://www.nt.tuwien.ac.at/research/rapid/
}

\begin{abstract}
Closed loop transmission diversity will be an important part of the enhancements of today's wireless technologies, e.g. in double-stream transmit antenna array (D-TxAA). Despite the promise of performance gains, closed loop beamforming will introduce an interference scenario in the downlink that cannot easily be handled by the conventional MMSE equalization approach. In this paper, we derive an interference aware MMSE equalizer that uses the information about the beamforming vectors. The proposed equalizer can be calculated by utilizing a virtual channel description incorporating the beamforming weights. To assess the performance, we performed fading simulations with a system level description of the transmission. The derived equalizer shows only a small increase in complexity compared to the conventional approach, but perfectly restores the orthogonality.
\end{abstract}

\section{INTRODUCTION}

It is well known that the performance of modern cellular WCDMA networks is limited by interference, more than by any other single effect. This will be even more challenging in the future due to the rising demand in high data rates as requested by multimedia applications. In order to satisfy these needs, it will be crucial to employ both, enhanced transmission strategies, as well as enhanced receiver structures.

In the last 20 years, a lot of research has been conducted in the area of multi-user techniques, lately also for multiantenna systems. It has to be noted that generally uplink and downlink scenarios have very different characteristics, such that they are likely to be further differentiated in future cellular systems. In particular, in the downlink each receiver needs to decode a single desired signal, while experiencing two types of interference. These interference types emerge from the serving Node-B (intracell interference) and from a few dominant neighbouring Node-Bs (intercell interference). In the uplink, on the other hand, the base station receiver has to decode all desired users in the cell, while having to suppress neighboring cell interference from many independent sources. A good overview over the different interference situations together with some well known solutions for it can be found in [1].

Due to the distinctive differences between the up- and downlink, many of the proposed enhancements so far treat those two scenarios separately. Loosely speaking, many approaches in the downlink focus on enhanced coding strategies at the base station in order to effectively orthogonalize the users, e.g. [2], or on receiver structures which try to cancel the interference from the neighboring base stations, e.g. [3]. Also in the $3 \mathrm{GPP}$ standardization process, interference aware receivers are investigated [4], because multiple-input multipleoutput (MIMO) systems are even more subject to interference limitations. However, despite the efforts in this field, today's receivers deployed in the user equipment still treat the interference as white Gaussian.

In this paper, we want to develop an interference aware space-time MMSE (ST-MMSE) equalizer for the downlink of transmit antenna array (TxAA) [5] MIMO high-speed downlink packet access (HSDPA), which utilizes the special structure of the intracell interference. By considering the beamforming vectors of the synchronously served users in the cell, the proposed MMSE equalizer is able to outperform the conventional MMSE equalizer. A system level description of the signal to interference ratio (SIR) is developed and used to assess the performance of our approach.

The paper is organized as follows: in Section II we discuss some details about the underlying TxAA transmission scheme. After that, we present the used system model and our proposed MMSE equalizer in Section III. System-level fading simulations showing the performance enhancements are presented in Section IV. Finally, our conclusions are given in Section V.

\section{TXAA}

High-speed downlink packet access, after its introduction in 3GPP Release 5 (see e.g. [6]), has been the key technology in providing high downlink data rates in existing WCDMA networks. Future enhancements of this transmission scheme are mainly based on MIMO enhancements, see [7], where many proposals have been investigated. Finally, during the second quarter of 2006, the radio access network (RAN) group decided in favor of the "Dual-Stream TxAA" (D-TxAA), which is a closed loop transmission scheme. Furthermore, it was agreed to focus on the deployment of two transmit antennas.

The D-TxAA mode allows for the transmission of two parallel transport blocks, which are coded, interleaved and spread as in the non-MIMO mode. However, when channel conditions are not favorable, either the mobile will suffer from the bad channel quality and thus report a low channel quality indicator (CQI) value, or the scheduler will decide on 


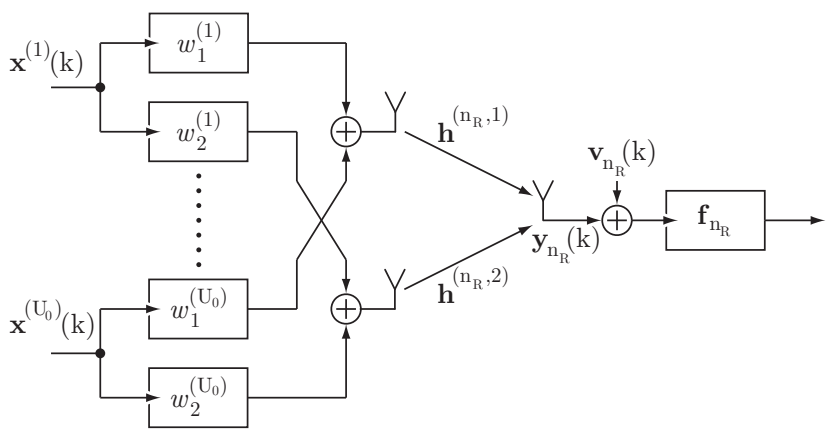

Fig. 1. Multiuser transmission in TxAA, for a total number $U_{0}$ of paralle served users, with one transmit antenna depicted. The transmission over the two available antennas is weighted by individual beamforming weights for each user.

a downgrade. Both will result in a single-stream transmission (hence only utilizing the primary transport block), very similar to the already known closed loop Mode 1 transmit diversity, as e.g. described in [5], which we conveniently call "TxAA" mode for HSDPA.

Regardless of the number of used streams, the precoding weights are determined from a quantized set by the user equipment (UE) to maximize the received signal-to-interference and noise ratio (SINR). Obviously, if more than one user is served, different sets of precoding weights may be requested from different users. The NodeB in addition to applying these sets furthermore has to signal them on a control channel to inform the UEs about the current set in use. Please note that TxAA, as a transmission strategy, works for an arbitrary number of receive antennas.

\section{SySTEM MODEL}

Within the sketched TxAA framework we now want to highlight some particularities that will lead to the formulation of our novel equalizer. Figure 1 shows the parallel transmission of the already spread/scrambled data, $\mathbf{x}^{(1)}(k), \ldots, \mathbf{x}^{\left(U_{0}\right)}(k)$, for $U_{0}$ users. Obviously, always the same two transmit antennas are used, but the served users can have different beamforming weights $\left(w_{1}^{(1)}, w_{2}^{(1)}\right), \ldots,\left(w_{1}^{\left(U_{0}\right)}, w_{2}^{\left(U_{0}\right)}\right)$ applied. The illustrated channel coefficients $\mathbf{h}^{\left(n_{R}, n_{T}\right)}, n_{T}=1,2$ are composed of the taps of the frequency selective channel between transmit antenna $n_{T}$ and receive antenna $n_{R}=1, \ldots, N_{R}$.

If the beamforming weights are incorporated in a so-called equivalent channel, the multiuser transmission of Figure 1 can be represented by $U_{0}$ virtual antennas, one for each active user, as illustrated in Figure 2. The resulting equivalent (virtual) channel between the virtual transmit antenna of user $u$ and the receive antenna $n_{R}$ is given by

$$
\tilde{\mathbf{h}}^{\left(u, n_{R}\right)}=w_{1}^{(u)} \mathbf{h}^{\left(n_{R}, 1\right)}+w_{2}^{(u)} \mathbf{h}^{\left(n_{R}, 2\right)} .
$$

From this description, it now can easily be seen that the intracell interference generated by the downlink transmission in a TxAA system is not only transmitted over the channel of the desired user. If we assume the desired user to be $u=1$, Figure 2 shows that all other active users with

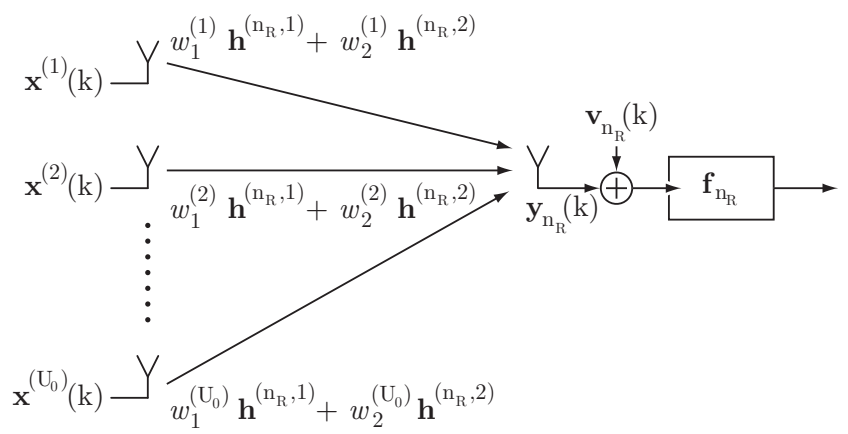

Fig. 2. Equivalent representation of the multiuser TxAA transmission in Figure 1.

different beamforming weights $\left(w_{1}^{(u)}, w_{2}^{(u)}\right)$ will generate an interference term that cannot be canceled out by a classical MMSE equalizer that is only matched to the channel of the desired user (determined by the beamforming weights $\left(w_{1}^{(1)}, w_{2}^{(1)}\right)$ in our example) and that considers all interference as additive white Gaussian noise.

\section{A. Enhanced ST-MMSE Equalizer}

With these details about the TxAA multiuser transmission in mind, we now want to sketch the important points in the derivation of an enhanced MMSE equalizer, being able to cancel out the intracell interference generated by users with different beamforming weights. Let the transmit chip vector of user $u$ at time instant $k$ be given by

$$
\mathbf{x}^{(u)}(k)=\left[x^{(u)}(k), \ldots, x^{(u)}(k-L-E+2)\right]^{\mathrm{T}},
$$

where $L$ and $E$ are the length of the channel impulse response and the equalizer span, respectively. The transmitted vectors $\mathbf{x}^{(u)}(k)$ of all $U_{0}$ users can be stacked, forming the overall transmit chip vector

$$
\mathbf{x}(k)=\left[\left(\mathbf{x}^{(1)}(k)\right)^{\mathrm{T}}, \ldots,\left(\mathbf{x}^{\left(U_{0}\right)}(k)\right)^{\mathrm{T}}\right]^{\mathrm{T}} .
$$

As already indicated, the frequency selective fading between the $U_{0}$ virtual transmit antennas and the considered receive antenna of the user equipment is modeled by $U_{0}$ band matrices of dimension $E \times L+E-1$. The channel matrix between the $u$-th virtual transmit antenna and the $n_{R}$-th receive antenna is given by

$$
\begin{aligned}
& \mathbf{H}^{\left(n_{R}, u\right)}= \\
& {\left[\begin{array}{cccc}
\tilde{h}^{\left(n_{R}, u\right)}(0) & \ldots & \tilde{h}^{\left(n_{R}, u\right)}(L-1) & 0 \\
\ddots & & \ddots & \\
0 & \tilde{h}^{\left(n_{R}, u\right)}(0) & \ldots & \tilde{h}^{\left(n_{R}, u\right)}(L-1)
\end{array}\right],}
\end{aligned}
$$

where the $\tilde{h}^{\left(n_{R}, u\right)}(l)$ represent the virtual channel impulse response of the $u$-th user to the $n_{R}$-th receive antenna (see Equation (1)) can be calculated as

$$
\tilde{h}^{\left(n_{R}, u\right)}(l)=w_{1}^{(u)} h^{\left(n_{R}, 1\right)}(l)+w_{2}^{(u)} h^{\left(n_{R}, 2\right)}(l) .
$$


Here, the channel coefficients $h^{\left(n_{R}, 1\right)}(l)$ and $h^{\left(n_{R}, 2\right)}(l)$ denote the coefficients from transmit antenna one and two, respectively. Note that these coefficients represent the actual physical channel and are therefore the same for all users. Accordingly, the received signal at receive antenna $n_{R}$ can be represented by

$$
\begin{aligned}
\mathbf{y}_{n_{R}}(k) & =\left[y_{n_{R}}(k), \ldots, y_{n_{R}}(k-E+1)\right]^{\mathrm{T}} \\
& =\sum_{u=1}^{U_{0}} \mathbf{H}^{\left(n_{R}, u\right)} \mathbf{x}^{(u)}(k)+\mathbf{v}_{n_{R}}(k),
\end{aligned}
$$

which can be reformulated to

$$
\begin{aligned}
\mathbf{y}_{n_{R}}(k) & =\left[\mathbf{H}^{\left(n_{R}, 1\right)}, \ldots, \mathbf{H}^{\left(n_{R}, U_{0}\right)}\right] \mathbf{x}(k)+\mathbf{v}_{n_{R}}(k) \\
& =\mathbf{H}^{\left(n_{R}\right)} \mathbf{x}(k)+\mathbf{v}_{n_{R}}(k) .
\end{aligned}
$$

Here, we introduced the channel matrix $\mathbf{H}^{\left(n_{R}\right)}$ of size $E \times$ $U_{0}(L+E-1)$, and the noise vector $\mathbf{v}_{n_{R}}(k)$ of length $E$. By using the stacked vectors

$$
\begin{aligned}
& \mathbf{y}(k)=\left[\left(\mathbf{y}_{1}(k)\right)^{\mathrm{T}}, \ldots,\left(\mathbf{y}_{N_{R}}(k)\right)^{\mathrm{T}}\right]^{\mathrm{T}}, \\
& \mathbf{v}(k)=\left[\left(\mathbf{v}_{1}(k)\right)^{\mathrm{T}}, \ldots,\left(\mathbf{v}_{N_{R}}(k)\right)^{\mathrm{T}}\right]^{\mathrm{T}},
\end{aligned}
$$

and the stacked channel matrix

$$
\mathbf{H}=\left[\left(\mathbf{H}^{(1)}\right)^{\mathrm{T}}, \ldots,\left(\mathbf{H}^{\left(N_{R}\right)}\right)^{\mathrm{T}}\right]^{\mathrm{T}},
$$

we obtain

$$
\mathbf{y}(k)=\mathbf{H s}(k)+\mathbf{v}(k),
$$

where $N_{R}$ denotes the total number of receive antennas at the user equipment of the desired user.

At the receiver, the transmitted chip sequence of the desired user $(u=1)$ is reconstructed using the equalization filter $\mathbf{f}$, as shown in Figure 1 and 2. The equalizer coefficients can be calculated by minimizing

$$
J(\mathbf{f})=\mathbb{E}\left\{\left|\mathbf{f}^{\mathrm{H}} \mathbf{y}(k)-x^{(1)}(k-d)\right|^{2}\right\},
$$

with $d$ denoting the equalizer delay, and

$$
\mathbf{f}=\left[\mathbf{f}_{1}^{\mathrm{T}}, \ldots, \mathbf{f}_{N_{R}}^{\mathrm{T}}\right]^{\mathrm{T}} \text {. }
$$

The minimization of the cost function yields the equalizer coefficients for the data stream of the first user:

$$
\mathbf{f}^{\mathrm{H}}=\sigma_{x}^{2}\left(\mathbf{H R}_{x x} \mathbf{H}^{\mathrm{H}}+\mathbf{R}_{v v}\right)^{-1} \mathbf{H e}_{d},
$$

where the matrices $\mathbf{R}_{x x}$ and $\mathbf{R}_{v v}$ denote the signal and noise covariance matrices, respectively. Furthermore, $\mathbf{e}_{d}$ is a zero vector with a one at position $d$, which specifies the delay of the equalized signal, and has to fulfill $d \geq L$. Finally, $\sigma_{x}^{2}$ denotes the variance of the (independently assumed) chips. The "classical" single user MMSE equalizer is included as special case in Equation (14), if $\mathbf{H}$ is chosen to be

$$
\mathbf{H}=\left[\left(\mathbf{H}^{(1,1)}\right)^{\mathrm{T}}, \ldots,\left(\mathbf{H}^{\left(N_{R}, 1\right)}\right)^{\mathrm{T}}\right]^{\mathrm{T}} .
$$

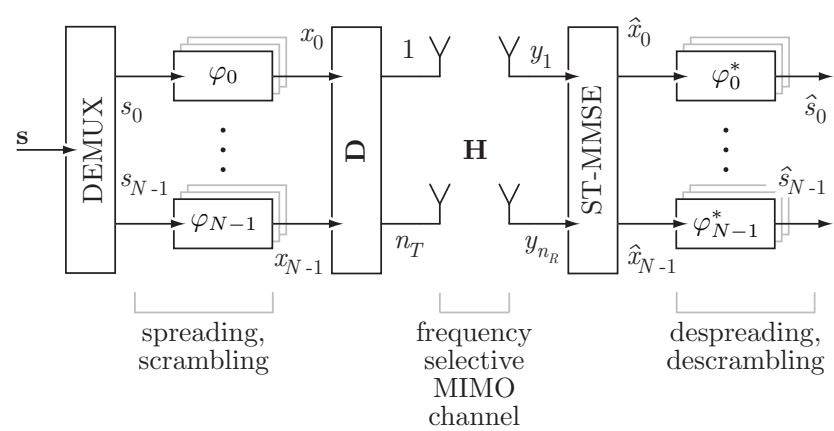

Fig. 3. Underlying concept of the system level link-measurement model, as introduced in [8].

\section{B. System Level Modeling}

To assess the performance of our proposed enhanced MMSE equalizer, we decided to use a system model based approach, which can be used to reveal the principal performance of a receiver in the context of a given transmission system without the need of detailed link level simulations. For our purposes in this paper, we adopted the system-level link measurement model, as introduced in [8]. This model is able to describe the post equalization and despreading SINR for arbitrary linear receivers in a multi-stream closed loop MIMO CDMA system.

Figure 3 illustrates the basic structure of the modeled system, where $s_{n}, n=0, \ldots, N-1$, denotes the symbol on the $n$-th of $N$ streams, $\varphi_{n}$ denotes the set of used spreading codes on stream $n$, and $\mathbf{D}$ represents the beamforming used at the transmitter.

With the mentioned link measurement model, the intracell interference generated by the desired user and all other active users can explicitly be given, i.e.

$$
P_{\text {intra }}=P_{1} \cdot \sum_{\substack{m=0 \\ m \neq d}}^{E+L-2}\left|\mathbf{f}^{\mathrm{T}} \boldsymbol{\gamma}_{00}^{m}\right|^{2}+\sum_{u=2}^{U_{0}} P_{u} \cdot \sum_{\substack{m=0 \\ m \neq d}}^{E+L-2}\left|\mathbf{f}^{\mathrm{T}} \boldsymbol{\gamma}_{u 0}^{m}\right|^{2},
$$

where, in contrast to [8], we chose $N=1$, since in TxAA there is only one transmitted data stream for each user. In Equation (16), we assumed (without loss of generality) that user $u=1$ is the desired user. Furthermore, $P_{u}$ denotes the total transmission power for user $u$ and $\gamma_{u 0}^{m}$ is the $m$-th column of the equivalent channel matrix of user $u$,

$$
\mathbf{H}^{(u)}=\left[\left(\mathbf{H}^{(1, u)}\right)^{\mathrm{T}}, \ldots,\left(\mathbf{H}^{\left(N_{R}, u\right)}\right)^{\mathrm{T}}\right]^{\mathrm{T}} .
$$

As described in [8], Equation (16) can be further simplified for system level simulations by taking the expectation over the used beamforming weights of the other users, i.e.

$$
\begin{aligned}
& P_{\text {intra }} \approx \\
& P_{1} \cdot \sum_{\substack{m=0 \\
m \neq d}}^{E+L-2}\left|\mathbf{f}^{\mathrm{T}} \boldsymbol{\gamma}_{00}^{m}\right|^{2}+\mathbb{E}_{w}\left\{\sum_{u=2}^{U_{0}} P_{u} \cdot \sum_{\substack{m=0 \\
m \neq d}}^{E+L-2}\left|\mathbf{f}^{\mathrm{T}} \boldsymbol{\gamma}_{u 0}^{m}\right|^{2}\right\},
\end{aligned}
$$


TABLE I

SIMULATION PARAMETERS FOR FIGURES 4 AND 5.

\begin{tabular}{|c|c|}
\hline Parameter & Value \\
\hline \hline \# simulated slots & 10000 \\
slot time & $2 / 3 \mathrm{~ms}$ \\
\# receive antennas $N_{R}$ & 2 \\
equalizer span $E$ & 30 \\
equalizer delay $d$ & 15 \\
beamforming delay & $3 \mathrm{slots}$ \\
mobile speed & $3 \mathrm{~km} / \mathrm{h}$ \\
channel profile & ITU PedA \\
\# active users $U_{0}$ & 4 \\
fading model & improved Zheng model \\
\hline
\end{tabular}

which, if equiprobable beamforming vectors are assumed, results in

$$
P_{1} \cdot \underbrace{\sum_{\substack{m=0 \\ m \neq d}}^{E+L-2}\left|\mathbf{f}^{\mathrm{T}} \boldsymbol{\gamma}_{00}^{m}\right|^{2}}_{f_{\text {self }}}+\sum_{u=2}^{U_{0}} P_{u} \cdot \underbrace{\frac{1}{\Omega} \sum_{b=1}^{\Omega} \sum_{\substack{m=0 \\ m \neq d}}^{E+L-2}\left|\mathbf{f}^{\mathrm{T}} \boldsymbol{\gamma}_{b 0}^{m}\right|^{2}}_{f_{\text {other }}},
$$

where $\Omega$ denotes the number of possible beamforming vectors $\left(w_{1}, w_{2}\right)$. The introduced terms $f_{\text {self }}$ and $f_{\text {other }}$ can be used to assess the ability of the equalizer, since $f_{\text {self }}$ describes the ability to cancel the intracell interference of the desired user itself, and $f_{\text {other }}$ describes the ability to suppress the intracell interference generated by other users.

\section{FADing Simulations}

With the intracell interference system level model from Section III-B, we now want to compare the performances of the classical and our proposed enhanced MMSE equalizer. In principle, the solution derived in Section III-A can be used regardless of the set of allowed beamforming vectors. In DTxAA for example, only four different beamforming vectors are possible, thus reducing the amount of uplink feedback to two bits. For our fading simulations, utilizing the system level model, we restricted ourselves to the TxAA case with the four beamforming vector possibilities as defined in [7].

Table I shows the settings of the simulation parameters for the Figures 4 and 5. We used a fading simulator, utilizing the improved Zheng model (see [9] and [10]) to generate the MIMO channel coefficients, where the channel profile was chosen according to the ITU-T recommendations [11]. The equalizer calculation (updated every slot), the beamforming selection, as well as the beamforming delay were considered in the simulator. For the receiver, we assumed perfect channel knowledge.

Figure 4 shows the mean value of $f_{\text {self }}$, averaged over the number of simulated slots. It can be observed that with increasing mean receive SINR, the equalizer is able to suppress the remaining own fraction of the intracell-interference after equalization. Subsequently, Figure 5 shows the applicable mean value of $f_{\text {other }}$ for the same simulation run. In contrast to

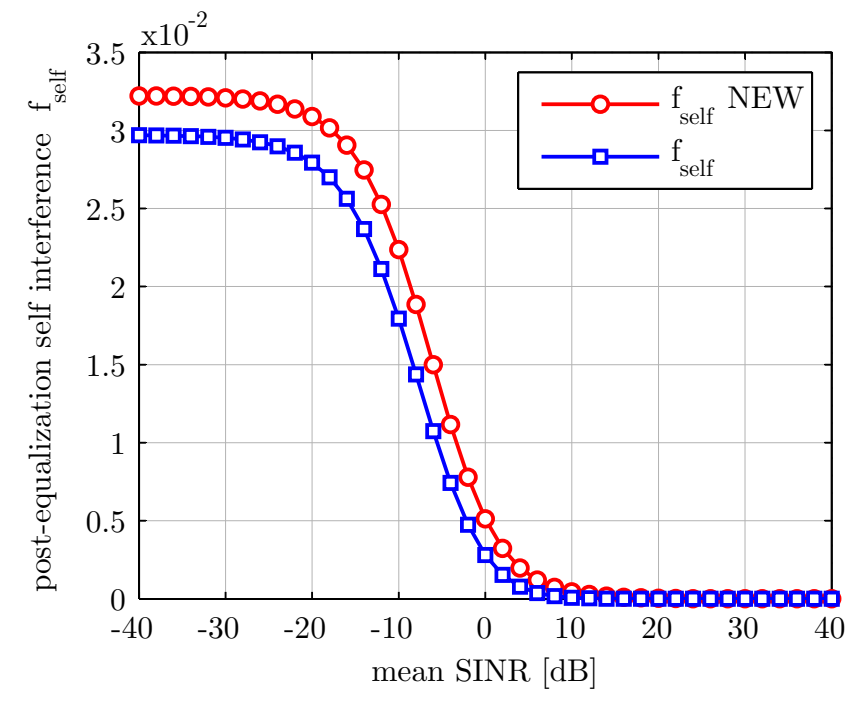

Fig. 4. Simulation results of mean $f_{\text {self }}$ (fraction of post-equalization intracell interference generated by the desired user, called "self-interference") for the ITU PedA channel profile.

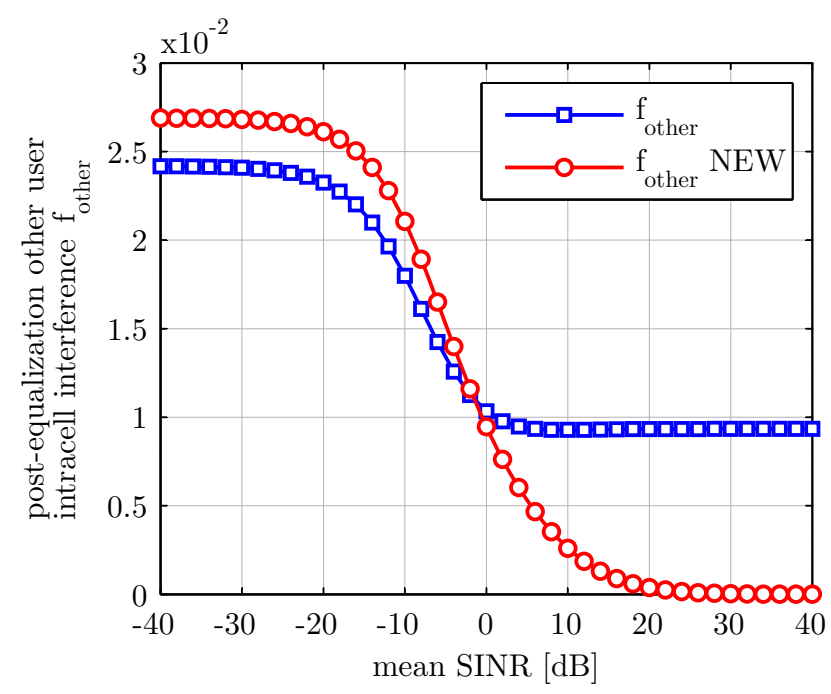

Fig. 5. Simulation results of mean $f_{\text {other }}$ (fraction of post-equalization intracell interference generated by other users) for the ITU PedA channel profile.

the previous figure, it can be seen that our proposed equalizer (denoted by "NEW") outperforms the classical equalizer for higher mean receive SINRs. In exchange, it sacrifices performance in the lower SINR regime, as it can be observed in Figure 4.

In case of the ITU VehA channel profile, the performance gain of our novel MMSE equalizer is even more considerable. Figures 6 and 7 show the post-equalization fractions of the intracell interference generated by the desired user and the other users, respectively. For these simulations, the same settings as in Table I were used, but the mobile speed was set to $120 \mathrm{~km} / \mathrm{h}$.

Figure 7 shows that the conventional MMSE equalizer even performs worse with increasing transmission power, but our 


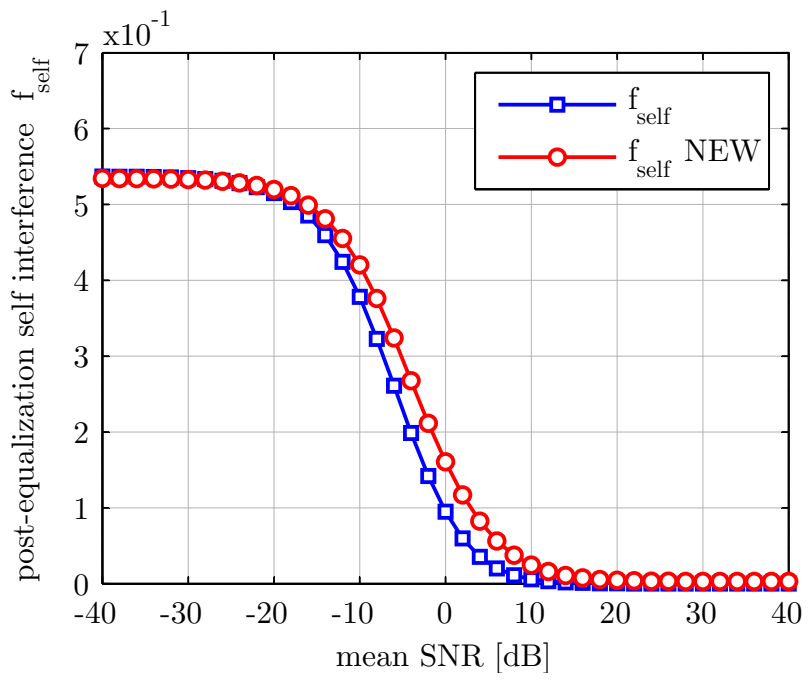

Fig. 6. Simulation results of mean $f_{\text {self }}$ (fraction of post-equalization intracel interference generated by the sired user, called "self-interference") for the ITU VehA channel profile.

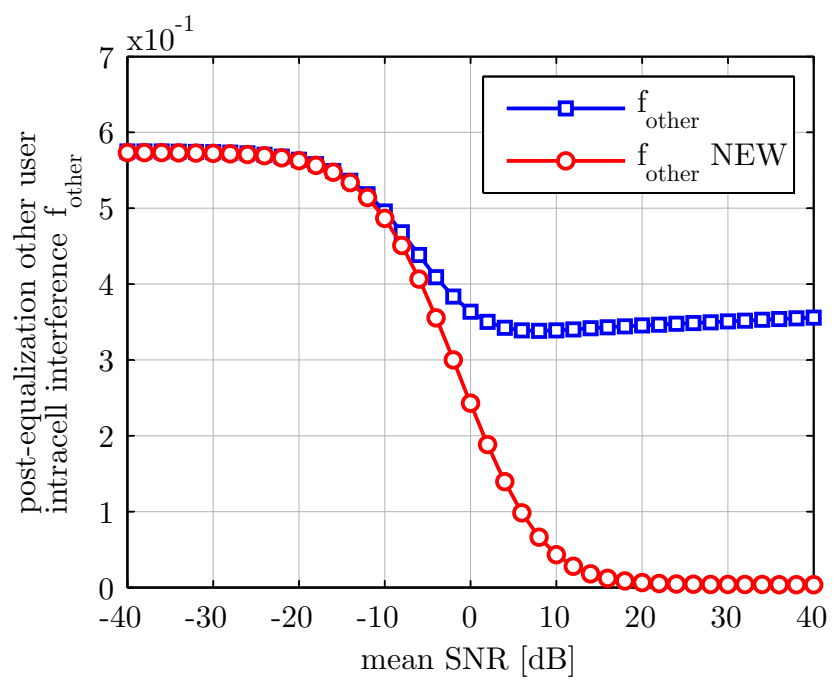

Fig. 7. Simulation results of mean $f_{\text {other }}$ (fraction of post-equalization intracell interference gerated by other users) for the ITU VehA channel profile.

proposed equalizer is able to suppress the interference to the full amount, just as in the PedA case. Interestingly, as illustrated in Figure 6, our equalizer shows nearly the same performance in the low SINR regime in terms of the postequalization self interference $f_{\text {self }}$. This is caused by the higher delay spread of the VehA profile in interaction with the limited equalizer span, where the achievable performance is limited due to our choice of $E=30$.

We also conducted simulations with different choices of the equalizer span which are omitted here. However, for higher equalizer spans, one would observe a flattened gradient of the post-equalization intracell interference caused by the other users, $f_{\text {other }}$. Accordingly, $f_{\text {other }}$ in Figure 7 would be monotonic decreasing for increasing mean receive SNR.

\section{CONCLUSIONS}

We proposed an MMSE equalizer for TxAA based MIMO transmission schemes that is effectively able to cancel the intracell interference, even in the case that other users, utilizing different beamforming vectors, are active. The derivation of the equalizer together with a short description of an appropriate system level model to assess its performance have been presented. Fading simulations showed the potential of the new equalizer to outperform the classical single user MMSE equalizer.

However, note that for the current proposal, the MMSE equalizer has to know all active beamforming weights. These weights can be obtained by monitoring the control channels of all users. Future research will investigate the performance of an equalizer that does not need this information. Furthermore, link-level simulations will show the performance in terms of throughput.

\section{ACKNOWLEDGEMENT}

This work was carried out within the ftw. project $\mathrm{C} 12$ and was funded by Kplus, Infineon Technologies, Mobilkom Austria AG, the University of Linz and the Christian Doppler Laboratory for Design Methodology of Signal Processing Algorithms. The authors would like to thank Maxime Guillaud, ftw. for continuous encouragement and support.

\section{REFERENCES}

[1] J. Andrews, "Interference cancellation for cellular systems: A contemporary overview," IEEE Wireless Commun. Mag., vol. 12, no. 2, pp. 19-29, Apr. 2005.

[2] H. Zhang and H. Dai, "Cochannel interference mitigation and cooperative processing in downlink multicell multiuser MIMO networks," EURASIP J. Wireless Commun. Netw., vol. 2, pp. 222-235, Dec. 2004

[3] F. Petre, M. Engels, A. Bourdoux, B. Gyselinckx, M. Moonen, and H. D. Man, "Extended MMSE receiver for multiuser interference rejection in multipath DS-CDMA channels," in Vehicular Technology Conference (VTC 1999). IEEE, Sep. 1999.

[4] Members of 3GPP, "Technical specification group radio access network; feasibility study on interference cancellation for UTRA FDD user equipment (UE)," 3GPP, Tech. Rep. 3GPP TR 25.963 Version 7.0.0, Apr. 2007.

[5] — "Technical specification group radio access network; physical layer procedures (FDD)," 3GPP, Tech. Rep. 3GPP TS 25.214 Version 7.5.0, May 2007.

[6] - "Technical specification group radio access network; UTRA high speed downlink packet access (HSDPA); overall description; stage 2,' 3GPP, Tech. Rep. 3GPP TS 25.308 Version 5.0.0, Sep. 2001.

[7] — , "Technical specification group radio access network; multiple input multiple output in UTRA," 3GPP, Tech. Rep. 3GPP TR 25.876 Version 7.0.0, Mar. 2007.

[8] M. Wrulich, I. Viering, S. Eder, and M. Rupp, "Efficient link measurement model for system-level evaluations of D-TxAA MIMO HSDPA," 2007, in preparation.

[9] Y. Zheng and C. Xiao, "Simulation models with correct statistical properties for rayleigh fading channels," IEEE Trans. Commun., vol. 51, no. 6, pp. 920-928, Jun. 2003.

[10] T. Zemen and C. Mecklenbräuker, "Time-variant channel estimation using discrete prolate spheroidal sequences," IEEE Trans. Signal Process., vol. 53, no. 9, pp. 3597-3607, Sep. 2005.

[11] "Recommendation ITU-R M.1225: Guidelines for evaluation of radio transmission technologies for IMT-2000,” Tech. Rep., 1997. 